\title{
Endocrine response and outcome in 14 cats with insulin resistance and acromegaly treated with stereotactic radiosurgery ( $17 \mathrm{~Gy}$ )
}

\author{
Maegan L. Watson-Skaggs, DVM ${ }^{1}$; Tracy L. Gieger, DVM ${ }^{1,2}$; Hiroto Yoshikawa, DVM, PhD ${ }^{1,2 *}$; Michael W. Nolan, DVM, PhD ${ }^{1,2}$ \\ 'Department of Clinical Sciences, College of Veterinary Medicine, North Carolina State University, Raleigh, NC \\ ${ }^{2}$ Comparative Medicine Institute, College of Veterinary Medicine, North Carolina State University, Raleigh, NC \\ *Corresponding author: Dr. Yoshikawa (hyoshik@ncsu.edu)
}

\section{OBJECTIVE}

To describe clinical outcomes in cats with insulin resistance and acromegaly treated with stereotactic radiosurgery (SRS).

\author{
ANIMALS \\ 14 client-owned cats.
}

\section{PROCEDURES}

Medical records of cats with insulin resistance and acromegaly treated with SRS (I7 Gy) between August 2013 and November 2019 at a single institution were reviewed. Kaplan-Meier analysis was used to evaluate overall survival time.

\section{RESULTS}

Acute adverse effects of SRS included somnolence $(n=2)$ and alopecia $(I)$. Delayed adverse effects of SRS included unspecified neurologic complications $(\mathrm{n}=\mathrm{I} ; 48 \mathrm{I}$ days), seizures $(\mathrm{I} ; \mathrm{I}, 54 \mathrm{I}$ days), and hypothyroidism $(\mathrm{I} ; 64$ days). Exogenous insulin requirements decreased in 10 of the 14 cats, with a median time to lowest insulin dose of 399 days (range, 42 to 879 days). Complete diabetic remission was achieved in 3 cats. The median overall survival time was 74 I days ( $95 \%$ Cl, 353 to I, I29 days). Six cats were still alive at the end of the study period, with a median follow-up time of 725 days. In 7 of the 8 cats that had died, death was presumptively attributed to acromegaly owing to continued insulin resistance, organ failure, or altered neurologic status.

\section{CLINICAL RELEVANCE}

The SRS protocol was well tolerated and associated with survival times similar to those reported previously. Most cats had decreased exogenous insulin requirements after SRS. Latency to an endocrine response was highly variable, emphasizing the need for careful ongoing diabetic monitoring of acromegalic cats after pituitary gland irradiation.

\begin{abstract}
A cromegaly results from a functional adenoma of the pituitary gland adenohypophysis that oversecretes growth hormone, with resulting subsequent excess hepatic production of insulin-like growth factor 1 (IGF-1). ${ }^{1-4}$ This hormonal excess leads to clinical signs of acromegaly, including one or more of the following: large body size, broad facial features, prognathia inferior, organomegaly, plantigrade stance, enlarged paws, heart murmur, and respiratory stridor. ${ }^{1-3}$ The most common clinical manifestation of acromegaly in cats is insulin-resistant diabetes mellitus. ${ }^{2}$ Notably, cats with acromegaly tend to gain weight, in contrast to the weight loss typically observed in cats with unregulated diabetes mellitus that do not have acromegaly. ${ }^{4}$ In addition, some cats with acromegaly may have neurologic abnormalities, including circling, ataxia, head pressing, mental dullness, personality changes, seizures, and blindness, due to the intracranial mass effect caused by pituitary gland enlargement. ${ }^{-8}$
\end{abstract}

No single test can definitively confirm the diagnosis of acromegaly in cats. Instead, the diagnosis is based on a combination of clinical signs, physical examination findings, and results of endocrine testing and cross-sectional imaging of the pituitary gland. Secretion of growth hormone is intermittent, and the serum half-life of growth hormone is short; additionally, no commercially available test for feline growth hormone concentration exists in the US. Thus, the screening test of choice is measurement of serum IGF-1 concentration, with high concentrations reported to have a sensitivity of $84 \%$ and specificity of $92 \%$ for the diagnosis of feline acromegaly. ${ }^{3}$ Results of cross-sectional imaging such as MRI or CT further support a diagnosis of acromegaly, and findings can include pituitary gland enlargement with suprasellar extension, frontal bone thickening, soft tissue accumulation in the nasal cavity (including the sinuses and pharynx), and mandibular prognathia. ${ }^{9,10}$ Whereas the normal height of the pituitary gland in cats is 
2.6 to $3.1 \mathrm{~mm}$ on transverse CT images and $3.2 \mathrm{~mm}$ $( \pm 0.4 \mathrm{~mm})$ on transverse postcontrast T1-weighted MRI images, ${ }^{11-13}$ in 1 publication, ${ }^{5}$ the pituitary gland height in acromegalic cats ranged from 4.1 to 18.6 mm on CT images.

Transsphenoidal hypophysectomy is the treatment of choice in human patients with acromegaly and results in normalization of serum IGF-1 concentrations in $60 \%$ to $95 \%$ of people, with a cure rate of $60 \%$ to $70 \%$ in patients with macroadenomas. ${ }^{14-17}$ For those patients requiring additional treatment, medical treatment is most often pursued next, with radiotherapy typically relegated to third-line treatment. ${ }^{14}$ Two recent publications ${ }^{18,19}$ describe transsphenoidal hypophysectomy in cats and report diabetes mellitus remission rates of $71 \%$ and $92 \%$. However, serious complications (hypothermia, hypotension, and airway obstruction) were common, and the periprocedural mortality rate reportedly ranged from $4 \%$ to $15 \% .^{18-22}$ Medical treatment consisting of somatostatin analogs, dopamine agonists, or growth hormone receptor antagonists in cats is also generally unrewarding and cost prohibitive. ${ }^{23-26}$ However, the use of both short- and long-acting pasireotide (a somatostatin analog with binding affinity for somatostatin receptor subtypes 1,3 , and 5) has been associated with decreased serum IGF-1 concentrations and increased insulin sensitivity in acromegalic cats. ${ }^{25,26}$ In 1 study, ${ }^{25}$ all acromegalic cats that received 3 consecutive doses of short-acting pasireotide had a decrease in serum IGF-1 concentration on day 5, compared with the concentration on day 1 . In addition, longacting pasireotide administered as once-monthly SC injections in a group of acromegalic cats resulted in a significant decrease in median IGF-1 concentration and median insulin resistance index. ${ }^{26}$ Unfortunately, treatment with pasireotide is often cost prohibitive in cats. Because publications exist documenting the efficacy and relative safety of radiotherapy as a treatment for acromegaly, it is currently considered by many to be the best available and most accessible treatment option for cats.

Treatment with conventionally fractionated radiotherapy (total dose, 37 to $54 \mathrm{~Gy}$ in fractions of 2.7 to $5.0 \mathrm{~Gy}$ each) has been associated with reduced insulin requirements in $55 \%$ to $100 \%$ of acromegalic cats..$^{5-7,27,28}$ Stereotactic radiosurgery (SRS) and stereotactic radiotherapy (SRT) have also been associated with favorable outcomes. Of 9 cats with unregulated diabetes mellitus that were treated with a modified radiosurgical technique (a single fraction of 15 or $20 \mathrm{~Gy}$ planned non-graphically and delivered as a single unmodulated arc), 5 had decreased insulin requirements after radiosurgery. ${ }^{29} \mathrm{~A}$ more recent study ${ }^{8}$ of fractionated SRT in 53 cats with acromegaly (most of whom were treated with 3 consecutive daily fractions of $8 \mathrm{~Gy}$ each) reported decreased insulin requirements in $95 \%$ of cats, with $32 \%$ of those cats having a permanent (62\%) or temporary $(38 \%)$ remission in their diabetes mellitus. Median overall survival time in that study was 1,072 days $(95 \% \mathrm{CI}$, 845 to 1,339 days).

One particular challenge with SRS and SRT for feline acromegaly is that the time to onset of an endocrinologic response is highly variable and often quite long. Although Wormhoudt et $\mathrm{al}^{8}$ reported that the median time to lowest insulin dose was 9.5 months for cats that underwent SRT, at least 1 cat in their case series had a documented response within a month of irradiation. Similarly, Mayer et $\mathrm{al}^{7}$ reported that insulin requirements began decreasing within the first 2 months after full-course radiotherapy in 2 of 6 diabetic cats. In a case report, Littler et $\mathrm{al}^{30}$ reported a hypoglycemic episode in a cat 2 months after a hypofractioned course of radiotherapy, which necessitated a decrease in the exogenous insulin dose. When conventionally fractionated radiotherapy is used in human patients (eg, for treatment of tumors that are large or in close proximity to optic pathways), hormonal normalization may not be achieved until 10 to 15 years after radiotherapy. ${ }^{14,31}$ However, hormonal normalization can occur as quickly as 54 to 66 months after SRS with the Gamma Knife procedure. ${ }^{31}$ With hopes that extreme hypofractionation may have a similar benefit for cats in terms of reducing latency to endocrinologic response, all acromegalic cats undergoing radiotherapy at our institution since 2013 have been treated with a novel SRS protocol under which $99 \%$ of the gross tumor volume receives $17 \mathrm{~Gy}$ with a simultaneous integrated boost of $\geq 20$ Gy centrally within the tumor. The objective of the retrospective study reported here was to report our early clinical experiences with this novel SRS protocol in cats with insulin resistance and acromegaly.

\section{Materials and Methods}

\section{Selection criteria}

A single-arm, noncontrolled, observational retrospective study was performed. The radiation oncology patient database at the North Carolina State University College of Veterinary Medicine was searched to identify all cats that underwent SRS (17 Gy) between August 2013 and November 2019. Cats were eligible for inclusion in the study if a clinical diagnosis of acromegaly had been made on the basis of a high serum IGF-1 concentration ( $>92 \mathrm{nmol} / \mathrm{L}$ ) in combination with pituitary enlargement on crosssectional imaging. Alternatively, cats were included if they had clinical signs associated with acromegaly, which could include large body size, broad facial features, prognathia inferior, organomegaly, plantigrade stance, and enlarged paws. Cats were excluded if they did not have concurrent diabetes mellitus or did not require exogenous insulin.

\section{Data collection}

Information regarding signalment, results of cross-sectional imaging, radiation treatment planning, exogenous insulin requirements before and af- 
ter SRS, adverse events, and overall survival time was recorded after reviewing medical records and followup information obtained from referring veterinarians and clients. Acute adverse events were defined as treatment-associated complications occurring within 90 days of SRS. ${ }^{32}$ Late (delayed) adverse events were defined as treatment-associated complications occurring $>6$ months after SRS. ${ }^{32}$ Recommended screening tests prior to SRS included CBC, serum biochemistry profile, urinalysis, urine culture and susceptibility testing, serum IGF-1 concentration, thoracic radiography, abdominal ultrasonography, echocardiography, MRI, and total T4 concentration. The maximum height of the pituitary gland was measured on a postcontrast transverse CT images with electronic calipers by a single investigator (Supplementary Table SI). Pituitary gland height was included for informational purposes to further document the range of sizes seen in cats with acromegaly and was not used as an inclusion criterion. The height of the brain was obtained on the same transverse image; bones of the skull were not included in that measurement.

\section{Cross sectional imaging}

MRI was performed with a 1.5-T magnet (Magnetom Symphony; Siemens Medical Equipment Ltd) prior to October 2018 and a 3.0-T magnet (Magnetom Skyra; Siemens Medical Equipment Ltd) after October 2018. MRI images were obtained without the use of immobilization devices. The data set was acquired with a slice thickness of $2 \mathrm{~mm}$ or thinner. At a minimum, sequences obtained consisted of T2-weighted images in the transverse plane, FLAIR images, and T1-weighted images in the transverse, dorsal, and sagittal planes before and after IV gadolinium $(0.2 \mathrm{~mL} / \mathrm{kg})$ administration.

CT simulation scans were acquired in all cases prior to treatment (Somatom Perspective; Siemens Medical Equipment Ltd). At the time of CT simulation, cats were anesthetized and positioned in a bite block system as described by Harmon et al. ${ }^{33} \mathrm{~A}$ transverse multislice dataset was acquired before and after IV iohexol ( $2 \mathrm{mg} / \mathrm{kg}$ ) administration and reconstructed into 0.75 - and $1.0-\mathrm{mm}$ transverse sequences.

\section{Radiation treatment planning}

CT and MRI images were imported into the treatment planning system (Varian Eclipse version 11.2; Varian Medical Systems Inc), and MRI images were manually coregistered with CT images to guide tumor contour definition and contouring of the optic chiasm. ${ }^{34}$ Although pituitary gland masses were easily visible on postcontrast CT images, our institutional standard was to use both CT and MRI images for contouring the gross tumor volume for SRS. This was done because using MRI images for delineation of canine pituitary gland masses has been reported to yield larger volumes, compared with volumes obtained using CT images. ${ }^{35}$ Gross tumor volume was manually segmented as the entire pituitary gland.
Certain organs at risk were also contoured with postcontrast CT images and included brain, ear canals and cochleae, skin, palatine mucosa, bones, eyes, ocular lenses, pharynx, and esophagus. Gross tumor volume was removed from the normal brain contour for planning purposes. No expansions were applied to the tumor contour; thus, the planning target volume (PTV) was equivalent to the gross tumor volume. Radiation treatment plans were then constructed with inverse- or forward-planning techniques (on the basis of the prescriber's preference) to meet the planning objectives. If both intensity-modulated radiotherapy and nonmodulated radiotherapy plans were created, the plan with a more conformal dose and steeper dose gradient was used for treatment. All plans used a single isocenter and were created with 6-mV x-ray beams in a coplanar arrangement. The modulated plans were delivered at $600 \mathrm{MU} / \mathrm{min}$. When nonmodulated radiotherapy plans were created, multiple static gantry angles were used with multileaf collimator-fitted fields and a $0-\mathrm{mm}$ margin. The SRS $(1,000$ $\mathrm{MU} / \mathrm{min}$ ) function of the linear accelerator was used to deliver nonmodulated radiotherapy plans.

The intent for each plan was to deliver $100 \%$ of the radiation prescription (17 Gy) to $99 \%$ of the PTV with an intentional simultaneous integrated boost of 20 to 24 Gy delivered centrally within the PTV. The boost was achieved by creating a $1-$ to $2-\mathrm{mm}$ internal margin from the gross tumor volume in modulated plans. In nonmodulated plans, the collimator was fit to the external margin of the target with no additional margin for penumbra. The nonmodulated plans were then normalized so that $99 \%$ of the PTV received $100 \%$ of the prescription dose (17 Gy), which in turn created a natural centrally located boost. Dose-volume constraints for organs at risk were as previously reported for SRS of canine pituitary gland masses. ${ }^{35}$ These included $<1 \mathrm{~cm}^{3}$ of brain receiving $>16 \mathrm{~Gy}$ and dose falling to $14.4 \mathrm{~Gy}$ within $2 \mathrm{~mm}$ of the PTV's edge (brain - [PTV $+2 \mathrm{~mm}]$ ). The dose constraint goal for the optic chiasm was a maximum point dose of $14 \mathrm{~Gy}$.

Individual plan quality assurance was performed prior to treatment and was reviewed by a medical physicist. For intensity-modulated radiotherapy plans, quality assurance was performed with either a MapCheck2 device (Sun Nuclear Corp) or Varian Portal Dosimetry system (Varian Medical Systems Inc), an electronic portal imaging device on the linear accelerator. Quality-assurance software (RadCalc version 6.3; LifeLine Software Inc) was used as a second check of monitor unit calculations for nonmodulated radiotherapy plans. A passing quality-assurance score was defined as a minimum of $95 \% \gamma$ for a $3-\mathrm{mm}$ distance to agreement and $\mathrm{a} \leq 3 \%$ absolute dose difference for intensity-modulated radiotherapy plans and $<5 \%$ difference in monitor unit calculations for nonmodulated plans.

The following data were collected from the treatment planning system: dose to $99 \%$ of PTV; dose to 
95\% of PTV; near minimum (D98\%) and near maximum (D2\%) dose of PTV; dose to $50 \%$ of PTV; near minimum (D98\%) and near maximum (D2\%) dose to brain; volume of brain receiving 4,8 , and $10 \mathrm{~Gy}$; dose to $1 \mathrm{~cm}^{3}$ of brain; dose to $0.1 \mathrm{~cm}^{3}$ of brain - (PTV + $2 \mathrm{~mm}$ ); optic chiasm maximum point dose; and ears maximum point dose.

Conformity index and gradient index were retrospectively calculated as described by Paddick and Lippitz. ${ }^{36}$ The conformity and gradient indices were not used during treatment plan evaluation or approval and are reported to ensure completeness in reporting.

\section{Radiation treatment}

All treatments were delivered under general anesthesia within 2 weeks of CT simulation. Anesthesia protocols were not standardized, but all cats had IV catheters placed prior to anesthesia, and anesthetic protocols included injectable premedications, IV anesthetic drugs, endotracheal intubation, and maintenance of anesthesia with isoflurane gas. Clients were instructed to have the cat fasted and to administer half of the cat's usual insulin dose on the morning of SRS. Once anesthetized, cats were positioned in the same immobilization devices used for CT simulation. Accurate and precise patient positioning was achieved via image verification with cone-beam CT images, compared with simulation CT images by matching bones of the skull. A robotic couch top (Protura robotic patient positioning system; Civco Systems) with the ability to move with 6 degrees of freedom was used to make position changes as needed for the match. Therapy was delivered by use of a linear accelerator (Novalis Tx Technology) with a high-definition (2.5mm centrally located, 120-leaf) multileaf collimator.

\section{Follow-up}

Follow-up recommendations were not standard at our institution prior to December 2015. After that, we implemented the following follow-up recommendations: a complete blood glucose concentration curve 3 weeks after SRS, then monthly for the first 6 months after treatment; diabetes mellitus and adjustments in insulin dose to be managed by the referring veterinarian or an internal medicine specialist; recheck echocardiography 6 months after SRS; and thyroid function testing 3 and 6 months after SRS, then every 6 months thereafter.

\section{Statistical analysis}

Kaplan-Meier survival analysis (Prism version 8.1.2 for Windows; GraphPad Software) was performed. Overall survival time was defined as the number of days after SRS until death from any cause. Cats were censored from overall survival time analysis if they were still alive at the time of data analysis or lost to follow-up. In those cases, the date of the last patient follow-up was used for the overall survival time analysis.

\section{Results}

\section{Patient population}

Fifteen cats underwent SRS during the study period, but 1 cat was excluded because it had a pituitary gland tumor treated with SRS but did not have diabetes mellitus. For the cat that was excluded, presenting clinical signs included abnormal behavior described as staring into space and a lack of responsiveness to the owner. The remaining 14 cats met the criteria for inclusion in the study.

Individual patient information for the 14 treated cats was summarized (Supplementary Table S1). Hypertrophic cardiomyopathy was diagnosed by means of echocardiography in 6 cats $(5$ cats with a heart murmur and 1 cat with no murmur); 4 cats had normal echocardiographic findings. All cats with hypertrophic cardiomyopathy were reportedly mildly affected, and none required medical treatment. Three cats had evidence of both chronic kidney disease and hypertrophic cardiomyopathy.

MRI was performed in 12 of the 14 cats, and all 14 cats underwent CT simulation for planning purposes. One cat had a well-delineated pituitary gland located relatively far from the optic apparatus on CT images, so MRI was not performed. The reason for not performing MRI in the second cat was not recorded in the medical record.

In all cases, SRS was chosen by the owner after consultation with at least 1 American College of Veterinary Radiology board-certified radiation oncologist. All owners consented to treatment.

\section{SRS plans and dosimetric data}

All 14 cats were treated with the intended SRS protocol. Stereotactic radiosurgery plans were developed with inverse-planning (intensity-modulated radiotherapy; $\mathrm{n}=11$ ) or forward-planning (nonmodulated radiotherapy; 3) techniques. The most common beam arrangement was 7 coplanar beams (range, 5 to 11 beams). Mean and median doses to $99 \%$ of the PTV were 17.0 and 17.0 Gy, respectively (range, 17.0 to $17.1 \mathrm{~Gy}$ ). Dose statistics for the PTV and organs at risk were collected from the treatment planning software (Supplementary Table S2). Tumor coverage was prioritized over dose constraints for organs at risk. Conformity and gradient indices were retrospectively calculated.

\section{Follow-up and outcome}

Follow-up was performed by the primary care veterinarian or an internal medicine specialist for continued diabetic monitoring. However, the prescribed follow-up schedule was not strictly followed because of a lack of owner compliance, preference of individual veterinarians, or the cat was doing well clinically. Follow-up information gathered during the medical record review included a complete blood glucose concentration curve 3 weeks after SRS ( $\mathrm{n}=$ 7), monthly blood glucose concentration curves (5), 
recheck echocardiography 6 months after SRS (1 cat with stable hypertrophic cardiomyopathy), and thyroid function testing 3 and 6 months after SRS ( 5 and 4 cats, respectively). Two cats had serum fructosamine concentrations measured on a monthly basis. Eight cats had died by the time of data analysis, and the cause of death was attributed to acromegaly due to unregulated diabetes mellitus or other systemic effects associated with acromegaly in 7 of the 8 cats, including kidney failure $(\mathrm{n}=3)$, seizures (1), neurologic abnormalities and uncontrolled diabetes mellitus (1), repeated episodes of diabetic ketoacidosis (1), and heart failure (1). The remaining cat died suddenly 177 days after SRS after receiving 1 of 4 planned fractions of palliative-intent radiotherapy for a separate issue of chronic rhinitis. Six cats were still alive at the time of data analysis and were censored from the survival analysis; mean and median follow-up times for these censored cases were 730 days and 726 days, respectively (range, 213 to 1,462 days). Median overall survival time was 741 days (range, 101 to 1,540 days; $95 \%$ CI, 353 to 1,129 days; Figure I).

The only parameter readily available for comparison before and after radiotherapy for all cats in the study was exogenous insulin requirements. Ten of the 14 cats had a reduction in their insulin requirement after radiotherapy. Median time to the first required decrease in insulin requirement was 209 days (range, 42 to 714 days). Median time to the lowest insulin dose was 399 days (range, 42 to 879 days). Complete diabetes mellitus remission (ie, no longer requiring insulin) was achieved in 3 of these 10 cats. Diabetes remission was permanent in 2 of 3 cats; 1 cat was still alive 619 days after SRS, and the other died 291 days after SRS because of chronic kidney disease but did not require insulin at time of death. For the cat that achieved a temporary diabetes remission, remission started 600 days after SRS and lasted for 975 days. This cat was still

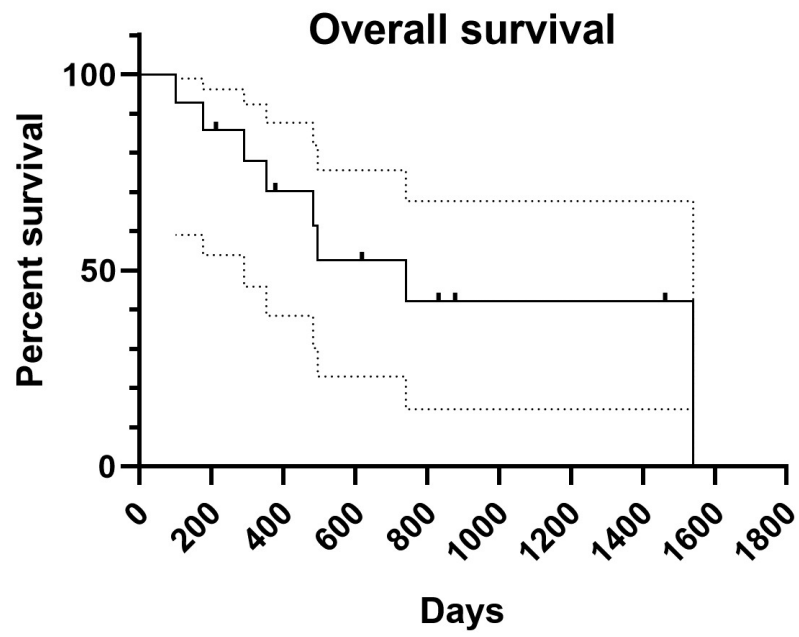

Figure I-Kaplan-Meier curve of overall survival time (solid line) for 14 cats with insulin resistance and acromegaly treated with stereotactic radiosurgery (17 Gy). Dotted lines represent the $95 \% \mathrm{Cl}$. Each step in the curve represents the death of I cat. Tick marks represent cats that were censored from the overall survival time analysis. alive at the end of data collection and censored from the survival analysis. All 4 cats that did not show an insulin response after SRS were dead at the time of data analysis; 2 were euthanized because of chronic kidney disease 101 and 496 days after SRS, 1 was euthanized because of repeated episodes of diabetic ketoacidosis 353 days after SRS, and 1 died suddenly during a course of palliative-intent radiotherapy for chronic rhinitis 177 days after SRS. This cat had follow-up CT for simulation prior to palliative-intent radiotherapy for chronic rhinitis; the pituitary gland measured $7.2 \mathrm{~mm}$ prior to SRS and $4.9 \mathrm{~mm}$ on recheck CT images. No other cats had follow-up CT.

\section{Radiation-associated adverse events}

Outcomes and adverse events for individual patients were summarized (Supplementary Table S3). Three cats reportedly had acute adverse events presumably related to SRS. Two cats were reported by their owners to have experienced somnolence with an onset of 5 and 6 days after SRS; in both cats, the somnolence resolved without any intervention. The owner of 1 cat reported temporary alopecia, but the location was not specified.

Three cats reportedly had late adverse events presumably related to SRS. One cat had unspecified neurologic signs 481 days after SRS, and a second cat had seizures 1,541 days after SRS. Neither cat was treated for their neurologic signs, and euthanasia was elected. In 1 cat, hypothyroidism was diagnosed 64 days after SRS. This cat had an unkempt haircoat, recent-onset pelvic limb weakness, and inappetence, which prompted thyroid function testing (T4 $<0.50 \mu \mathrm{g} / \mathrm{dL}$; reference range, 0.78 to $4.27 \mu \mathrm{g} / \mathrm{dL}$ ). The cat was subsequently treated with levothyroxine until the time of death 39 days later due to acute kidney injury. Necropsies were not performed on any of the cats.

\section{Discussion}

Our analysis of early clinical experiences with an SRS protocol for treatment of cats with insulin resistance and acromegaly suggested that the treatment was generally safe and that most cats (10/14) had a reduction in exogenous insulin requirements. However, the time to improved diabetic control was variable and often long. Additionally, for some cats, the duration of response was short.

Radiation treatments used in the present were planned with inverse- or forward-planning techniques, and our process for plan approval resulted in plans that were relatively homogeneous across all cats. The median conformity index of 0.73 indicated that plans were relatively conformal (a value of 1.0 would indicate perfect conformity). However, the gradient index suggested that dose falloff may not have been optimal. In a retrospective study, ${ }^{36}$ a gradient index $<$ 3.0 was considered ideal, whereas the median gradient index for the present study was 8.05. The seemingly high gradient index for this data set reflected 
the fact that the formula used to calculate gradient index skews toward high values when tumor volume is small. The pituitary gland can vary considerably in size in acromegalic cats, as demonstrated in a previous study, ${ }^{5}$ in which pituitary height ranged from 4.1 to $18.6 \mathrm{~mm}$, and in the present study, in which pituitary height ranged from 3.5 to $12.5 \mathrm{~mm}$. Thus, pituitary tumors in the cats of the present study were relatively small, compared with the size of many other solid tumors irradiated in veterinary and human oncology and with the size of pituitary glands in previous reports of acromegaly in cats. Regardless of the conformity and gradient indices, all plans did meet our predefined dose-volume objectives. This was demonstrated by a median D98\% (near minimum dose) of 17.3 Gy. Furthermore, the median D50\% (median dose) of nearly $20 \mathrm{~Gy}(19.8 \mathrm{~Gy})$ indicated that we met our goal of delivering a simultaneous integrated boost of 20 to $24 \mathrm{~Gy}$. These treatment plans met our dosimetric objectives and resulted in treatment safety and efficacy profiles similar to those in previous reports.

Acute morbidity in the present study was as expected. In a previous publication, ${ }^{8} 18 \%$ of cats (most of which were treated with an SRT protocol consisting of 3 fractions of 8 Gy each) experienced mild acute adverse events, including mental dullness, light sensitivity, and mydriasis. All of those cats were treated with prednisolone, and the adverse effects resolved. ${ }^{8}$ Two cats in this present series experienced somnolence within the week following SRS, and both cats improved without any medical intervention.

In relation to potential late treatment-induced morbidity, pituitary irradiation often involves unavoidable incidental irradiation of the optic apparatus. In humans, the optic nerve is potentially the most radiosensitive of the cranial nerves and is subject to injury after irradiation. ${ }^{34}$ Although the radiosensitivity of the feline optic apparatus is unknown, 1 cat in the previous publication ${ }^{34}$ describing SRT developed blindness without cataract formation 2 years after treatment. We did not systematically reassess cats described in the present study for changes in vision or ocular health; however, no cats were reported to have experienced blindness. However, 2 cats did develop neurologic signs and seizures long after SRS. Neither advanced imaging nor necropsy was pursued in those cats, and the most likely cause for these signs was thought to be either progressive tumor growth with a subsequent mass effect or radiation-induced brain necrosis. Another possible late adverse effect of pituitary irradiation is hypopituitarism, which can result in hypothyroidism, central diabetes insipidus, or both. Over $50 \%$ of people experience hypopituitarism after radiotherapy, with similar incidences for those undergoing conventional radiotherapy versus SRT. ${ }^{14}$ In the previously published study ${ }^{8}$ of 3 -fraction SRT protocol, $14 \%$ of cats developed hypothyroidism after undergoing SRT. Despite our recommendations for ongoing thyroid function testing after SRS, routine monitoring was not performed in the cats in the present study. Thus, it was impossible to document the true incidence of hypothyroidism in the study; however, 1 cat developed hypothyroidism 64 days after undergoing SRS.

The desired outcomes of prolonged survival time and improved diabetic control were achieved for most cats in the present study, with 10 of the 14 cats having documented endocrinologic responses. Furthermore, some of the cats died relatively soon after treatment, before they might have been expected to have a measurable benefit from SRS. In fact, of the 4 cats without a measurable endocrine response, 3 died before there had been sufficient time to fully understand their response status (ie, death within the first year after SRS in the context of a study population for which the median time to lowest insulin dose was 399 days after SRS). It is therefore possible that the response rate was underestimated in this case series. However, 2 of the 4 cats without a response died after the median time to a reduction of the exogenous insulin requirement (209 days), so it is also possible that they were true nonresponders. In addition to diabetic control, another potential response indicator could be changes in hormone concentration. However, growth hormone concentrations would be difficult to monitor because there are no commercially available assays and secretion is pulsatile. In previous studies, ${ }^{2,3}$ serum IGF-1 concentrations were not associated with clinical improvement or improved glycemic control after radiotherapy. On the other hand, improvements in IGF-1 concentrations have been shown to be associated with improved glycemic control and remission of diabetes mellitus after surgery. ${ }^{18,19}$ For these reasons, serum IGF-1 concentrations were not monitored after treatment in the present study, so no further conclusions can be drawn regarding IGF-1 concentrations and diabetic response after SRS.

In the present study, the first documented decrease in exogenous insulin requirements occurred at a median of 209 days after SRS, and the lowest exogenous insulin dose was reached at a median of 399 days (range, 42 to 879 days). Two cats required less insulin within the first 2 months after treatment, and 4 cats started requiring less insulin within 4 months after treatment. The other 6 cats with endocrine responses started requiring less insulin between 6 and 24 months after SRS. Because the time to onset of an endocrine response after radiotherapy is highly variable, cats should be routinely monitored after treatment, as their insulin requirement can change quickly and drastically. For example, one of the cats with a quick initial response went from requiring 10.5 to $12 \mathrm{U}$ of insulin twice daily to requiring only $5 \mathrm{U}$ of insulin twice daily 61 days after SRS. If a cat has responded to the treatment and is still receiving a high dose of insulin, there is a risk that the insulin overdose could cause a Somogyi effect, whereby exogenous insulin administration lowers the blood glucose concentration, triggering a release of hormones 
that in turn results in rebound hyperglycemia. ${ }^{37}$ Indeed, such a scenario may have contributed to the diabetic ketoacidosis that occurred in 1 cat in the current series.

To monitor for adverse effects and an endocrine response at our institution, we have implemented a standard set of follow-up recommendations after SRS. In particular, we advise that a specialist in internal medicine be engaged to assist with long-term management owing to the complicated nature of monitoring and managing insulin requirements in acromegalic cats, especially as they begin responding to radiotherapy. We generally recommend a blood glucose concentration curve be performed 3 weeks after SRS to establish a new posttreatment baseline and then monthly for the first 6 months, with an additional concentration curve performed 1 week after any change in insulin dose is made. This early and then frequent nature of testing is intended to optimize insulin dose management and reduce the risk of an insulin overdose resulting from the unpredictable nature of endocrine response times. As mentioned previously, hypothyroidism can be a late adverse effect, so we recommend rechecking serum thyroid hormone concentrations at 3 and 6 months after SRS and then every 6 months thereafter. If hypothyroidism is detected, treatment with levothyroxine is indicated. We also recommend recheck echocardiography 6 months after SRS, especially if cardiac changes were noted prior to treatment. This recommendation is supported on the basis of the finding that $13 \%$ of deaths in a previous study ${ }^{8}$ were due to heart failure or complications associated with hypertrophic cardiomyopathy.

Results of the present study indicated that this single-fraction SRS protocol was well tolerated by most cats with insulin resistance and acromegaly and provided a basis for developing protocols to monitor cats after treatment to optimize safety and tolerability. Longer follow-up of a large number of cases will enable a better definition of the overall endocrine response rate, response duration, and survival time associated with this protocol. What is clear from our findings is that highly variable endocrine response times remain problematic even with the type of extreme hypofractionation used to treat these cats. Future investigations of treatment protocols that use a combination of radiotherapy with medical therapies such as pasireotide, if this drug becomes more readily available and cost effective for use in veterinary medicine, might help improve endocrine control in cats that achieve only partial endocrine responses to radiotherapy. The potential additive effects of a pituitary tumor responding to both medical therapy and radiotherapy may allow for earlier or more predictable improvements in endocrine responses among acromegalic cats. In addition, radiotherapy protocols that allow for higher doses or dose intensities may offer an opportunity for quicker and more durable endocrine responses without losing the convenience of an SRT or SRS protocol. However, further investigation is needed.

\section{Acknowledgments}

No third-party funding or support was received in connection with this study or the writing or publication of the manuscript. The authors declare that there were no conflicts of interest.

\section{References}

1. Niessen SJM, Church DB, Forcada Y. Hypersomatotropism, acromegaly, and hyperadrenocorticism and feline diabetes mellitus. Vet Clin North Am Small Anim Pract. 2013;43(2):319-350.

2. Hurty CA, Flatland B. Feline acromegaly: a review of the syndrome. J Am Anim Hosp Assoc. 2005;41(5):292-297.

3. Berg RIM, Nelson RW, Feldman EC, Kass PH, Pollard R, Refsal KR. Serum insulin-like growth factor-I concentration in cats with diabetes mellitus and acromegaly. $J$ Vet Intern Med. 2007;21(5):892-898.

4. Lunn KF, Boston SE. Tumors of the endocrine system. In: Vail DM, Thamm DH, Liptak JM, eds. Withrow and MacEwen's Small Animal Clinical Oncology. 6th ed. Elsevier Saunders; 2020:569-571.

5. Brearley MJ, Polton GA, Littler RM, Niessen SJM. Coarse fractionated radiation therapy for pituitary tumours in cats: a retrospective study of 12 cases. Vet Comp Oncol. 2006;4(4):209-217.

6. Kaser-Hotz B, Rohrer CR, Stankeova S, Wergin M, Fidel J, Reusch C. Radiotherapy of pituitary tumours in five cats. $J$ Small Anim Pract. 2002;43(7):303-307.

7. Mayer MN, Greco DS, LaRue SM. Outcomes of pituitary tumor irradiation in cats. $J$ Vet Intern Med. 2006;20(5):1151-1154.

8. Wormhoudt TL, Boss M-K, Lunn K, et al. Stereotactic radiation therapy for the treatment of functional pituitary adenomas associated with feline acromegaly. J Vet Intern Med. 2018;32(4):1383-1391

9. Posch B, Dobson J, Herrtage M. Magnetic resonance imaging findings in 15 acromegalic cats. Vet Radiol Ultrasound. 2011;52(4):422-427.

10. Lamb CR, Ciasca TC, Mantis P, et al. Computed tomographic signs of acromegaly in 68 diabetic cats with hypersomatotropism. J Feline Med Surg. 2014;16(2):99-108.

11. Tyson R, Graham JP, Bermingham E, Randall S, Berry CR. Dynamic computed tomography of the normal feline hypophysis cerebri (glandula pituitaria). Vet Radiol Ultrasound. 2005;46(1):33-38.

12. Nadimi S, Molazem M, Jarolmasjed S, Esmaili Nejad MR. Volumetric evaluation of pituitary gland in dog and cat using computed tomography. Vet Res Forum. 2018;9(4):337-341.

13. Wallack ST, Wisner ER, Feldman EC. Mensuration of the pituitary gland from magnetic resonance images in 17 cats. Vet Radiol Ultrasound. 2003;44(3):278-282.

14. Melmed S, Colao A, Barkan A, et al. Guidelines for acromegaly management: an update. J Clin Endocrinol Metab. 2009;94(5):1509-1517.

15. Babu H, Ortega A, Nuno M, et al. Long-term endocrine outcomes following endoscopic endonasal transsphenoidal surgery for acromegaly and associated prognostic factors. Neurosurgery. 2017;81(2):357-366.

16. Shimon I, Cohen ZR, Ram Z, Hadani M. Transsphenoidal surgery for acromegaly: endocrinological follow-up of 98 patients. Neurosurgery. 2001;48(6):1239-1245.

17. Hazer DB, Isik S, Berker D, et al. Treatment of acromegaly by endoscopic transsphenoidal surgery: surgical experience in 214 cases and cure rates according to current consensus criteria.J Neurosurg. 2013;119(6):1467-1477.

18. van Bokhorst KL, Galac S, Kooistra HS, et al. Evaluation of hypophysectomy for treatment of hypersomatotropism in 25 cats. J Vet Intern Med. 2021;35(2):834-842.

19. Fenn J, Kenny PJ, Scudder CJ, et al. Efficacy of hypophysectomy for the treatment of hypersomatotropism-induced diabetes mellitus in 68 cats. J Vet Intern Med. 2021;35(2):823-833.

20. Neilson DM, Viscasillas J, Alibhai HI, Kenny PJ, Niessen SJ, Sanchis-Mora S. Anaesthetic management and complications 
during hypophysectomy in 37 cats with acromegaly. J Feline Med Surg. 2019;21(4):347-352.

21. Meij BP, Auriemma E, Grinwis G, Buijtels JJ, Kooistra HS Successful treatment of acromegaly in a diabetic cat with transsphenoidal hypophysectomy. J Feline Med Surg. 2010;12(5):406-410.

22. Blois SL, Holmberg DL. Cryohypophysectomy used in the treatment of a case of feline acromegaly. J Small Anim Pract. 2008;49(11):596-600.

23. Slingerland LI, Voorhout G, Rijnberk A, Kooistra HS. Growth hormone excess and the effect of octreotide in cats with diabetes mellitus. Domest Anim Endocrinol. 2008;35(4):352-361.

24. Abraham LA, Helmond SE, Mitten RW, Charles JA, Holloway SA. Treatment of an acromegalic cat with the dopamine agonist L-deprenyl. Aust Vet J. 2002;80(8):479-483.

25. Scudder CJ, Gostelow R, Forcada Y, Schmid HA, Church D, Niessen SJ. Pasireotide for the medical management of feline hypersomatotropism. J Vet Intern Med. 2015;29(4):10741080.

26. Gostelow R, Scudder C, Keyte S, et al. Pasireotide long-acting release treatment for diabetic cats with underlying hypersomatotropism. J Vet Intern Med. 2017;31(2):355-364.

27. Goossens MM, Feldman EC, Nelson RW, et al. Cobalt 60 irradiation of pituitary gland tumors in three cats with acromegaly. J Am Vet Med Assoc. 1998;213(3):374-376.

28. Peterson ME, Taylor RS, Greco DS, et al. Acromegaly in 14 cats. J Vet Intern Med. 1990;4(4):192-201.

29. Sellon RK, Fidel J, Houston R, Gavin PR. Linear-accelerator-based modified radiosurgical treatment of pituitary tumors in cats: 11 cases (1997-2008). J Vet Intern Med. 2009;23(5):1038-1044.

30. Littler RM, Polton GA, Brearley MJ. Resolution of diabetes mellitus but no acromegaly in a cat with pituitary macroade- noma treated with hypofractionated radiation. J Small Anim Pract. 2006;47(7):392-395.

31. Jezková J, Marek J, Hána V, et al. Gamma knife radiosurgery for acromegaly-long-term experience. Clin Endocrinol (Oxf). 2006;64(5):588-595.

32. Larue SM, Gordon, IK. Radiation oncology. In: Vail DM, Thamm DH, Liptak JM, eds. Withrow and MacEwen's Small Animal Clinical Oncology. 6th ed. Elsevier Saunders; 2020:212-213.

33. Harmon J, Van Ufflen D, Larue S. Assessment of a radiotherapy patient cranial immobilization device using daily on-board kilovoltage imaging. Vet Radiol Ultrasound. 2009;50(2):230-234.

34. Nolan MW, Randall EK, LaRue SM, Lunn KF, Stewart KF, Kraft SL. Accuracy of CT and MRI for contouring the feline optic apparatus for radiation therapy planning. Vet Radiol Ultrasound. 2013;54(5):560-566

35. Gieger TL, Nolan MW. Treatment outcomes and target delineation utilizing CT and MRI in 13 dogs treated with a uniform stereotactic radiation therapy protocol (16 Gy single fraction) for pituitary masses: (2014-2017). Vet Comp Oncol. 2021;19(1):17-24.

36. Paddick I, Lippitz B. A simple dose gradient measurement tool to complement the conformity index. J Neurosurg. 2006;105(suppl):194-201.

37. Roomp K, Rand J. Rebound hyperglycemia in diabetic cats. $J$ Feline Med Surg. 2016;18(8):587-596.

\section{Supplementary Materials}

Supplementary materials are posted online at the journal website: avmajournals.avma.org 